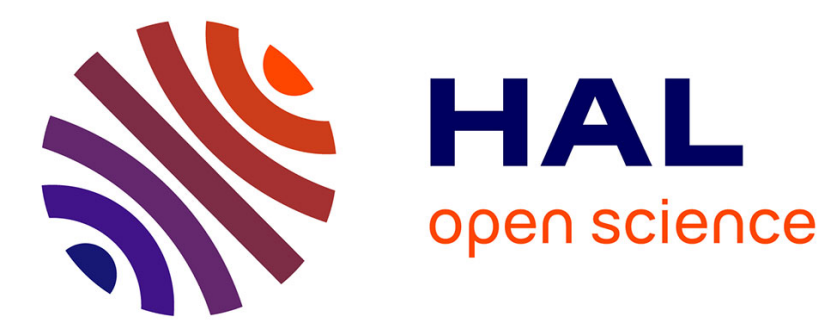

\title{
A Multifamily GLRT for CFAR Detection of Signals in a Union of Subspaces
}

\author{
François-Xavier Socheleau
}

\section{To cite this version:}

François-Xavier Socheleau. A Multifamily GLRT for CFAR Detection of Signals in a Union of Subspaces. IEEE Signal Processing Letters, 2020, 27, pp.2104-2108. 10.1109/LSP.2020.3039761 . hal03070891

\section{HAL Id: hal-03070891 \\ https://hal.science/hal-03070891}

Submitted on 16 Dec 2020

HAL is a multi-disciplinary open access archive for the deposit and dissemination of scientific research documents, whether they are published or not. The documents may come from teaching and research institutions in France or abroad, or from public or private research centers.
L'archive ouverte pluridisciplinaire HAL, est destinée au dépôt et à la diffusion de documents scientifiques de niveau recherche, publiés ou non, émanant des établissements d'enseignement et de recherche français ou étrangers, des laboratoires publics ou privés. 


\title{
A Multifamily GLRT for CFAR Detection of Signals in a Union of Subspaces
}

\author{
Francois-Xavier Socheleau, Senior Member, IEEE
}

\begin{abstract}
We consider the problem of detecting an unknown signal that lies in a union of subspaces (UoS) and that is observed in additive white Gaussian noise with unknown variance. The main contribution of this paper is the derivation of a detector that can accommodate a union made of nested subspaces. This detector includes the generalized likelihood ratio test (GLRT) as a special case when the subspace dimensions are all identical. It relies on the framework of multifamily likelihood ratio tests (MFLRT) and is shown by numerical examples to achieve better performance than existing detectors.
\end{abstract}

Index Terms-Multifamily likelihood ratio test, nested models, subspace detection.

\section{INTRODUCTION}

Testing whether a signal lies within a known subspace is a well-studied problem in the framework of matched-subspace detectors [1]-[6]. However, in practice, signals are often generated by multimodal processes so that there is not one but several possible subspace models. Among the set of possible models, the active subspace that generated the observed signal may be unknown a priori. This kind of signals complies with what is referred to as the UoS model. More precisely, $\mathbf{x} \in \mathbb{R}^{N}$ is an unknown signal belonging to some union of $M$ known subspaces, i.e., $\mathbf{x} \in \bigcup_{i=1}^{M} \mathcal{S}_{i}$, if and only if there exists $i_{0}$ such that $\mathbf{x} \in S_{i_{0}}$ [7]. In other words, $\mathbf{x}$ belongs to one of the subspace $\mathcal{S}_{i}$, but we do not know a priori to which one. UoS examples include signals with unknown spectral support [7], spectral signatures of radar targets [8] or sparse representations [9].

Signal detection under the UoS model has been partly addressed in the literature in the specific case where all subspaces are distinct, i. e., there are no subspaces in the union such that $\mathcal{S}_{i} \subset \mathcal{S}_{\ell}$, for $i \neq \ell$ [8]-[10]. All these works resort to the GLRT and estimate the active subspace index $i_{0}$ as the one that maximizes the likelihood functions. By assuming distinct subspaces, these methods fail to handle nested models properly. In our UoS context, nesting occurs when the signal can be expressed as the sum of an unknown number of basis vectors so that $\mathcal{S}_{i} \subset \mathcal{S}_{\ell}$, for $1 \leq i<\ell \leq M$. Examples include signals of unknown duration, periodic signal with an unknown number of harmonics, signals transmitted through an unknown multipath channel, etc. In that case, the GLRT always chooses the subspace model with the largest dimension leading to possible detection losses. More generally, problems arise with the GLRT when subspaces are of different dimensions and are not pairwise disjoint.

F.-X. Socheleau is with IMT-Atlantique, Lab-STICC, France (e-mail: \{fx.socheleau\}@imt-atlantique.fr).
In [11], the MFLRT has been introduced as a general solution to accommodate nested signal models. The idea is to add a penalty term to the GLR statistic to counteract its tendency to increase with the model order. More recently, a new normalizing transformation has been introduced in [12]. It generalizes the results of [11] and relies on the Legendre transform of the cumulant generating function (LT-CGF) of the test statistic. Although effective for various applications [13], [14], these results are not directly applicable to our context since the GLR statistic involved in our problem does not satisfy the required properties.

The main contribution of this letter is to adapt the LTCGF framework to the detection of an unknown signal in a UoS, with possibly overlapping or nested subspaces. The additive noise is assumed to be white Gaussian with unknown variance. The proposed detector is obtained by establishing a convergence result on the LT-CGF of a Fisher-z random variable. This approach generalizes the standard GLRT under the UoS model and includes it as a special case when the subspace dimensions are all identical. The paper is organized as follows. Sec. II is devoted to the problem formulation. The derivation of the Multifamily CFAR GLRT is presented in Sec. III. Numerical results are provided in Sec. IV, followed by conclusions in Sec. V.

Notation: We denote by $X_{n} \stackrel{d}{\rightarrow} X$ the convergence in distribution as $n \rightarrow+\infty$. $O(\cdot), O_{p}(\cdot)$ and $o_{p}(\cdot)$ designate the usual asymptotic notations for non-random and random variables, respectively [15, Ch. 14]. That is, $a_{n}=O\left(b_{n}\right)$ if the ratio $a_{n} / b_{n}$ is bounded for large $n, X_{n}=O_{p}\left(b_{n}\right)$ if the random sequence $X_{n} / b_{n}$ is stochastically bounded and $X_{n}=o_{p}(1)$ if $X_{n}$ converges to zero in probability. Finally, $f^{(m)}(x)$ denotes the $m$-th derivative of $f$ with respect to $x$.

\section{PROBLEM FORMULATION}

Let $\mathbf{x} \in \bigcup_{i=1}^{M} \mathcal{S}_{i}$, where $\mathcal{S}_{i}$ are subspaces of $\mathbb{R}^{N}$. No particular assumption is made with respect to the intersection of the subspaces and we do not assume all subspaces to have the same dimension but simply that $n_{i}=\operatorname{dim}\left(\mathcal{S}_{i}\right)<N, \forall i$. The detection problem addressed in this paper can be stated as

$$
\begin{cases}\mathcal{H}_{0}: & \mathbf{y}=\mathbf{w} \\ \mathcal{H}_{1}: & \mathbf{y}=\mathbf{x}+\mathbf{w}\end{cases}
$$

where $\mathbf{y}$ is the observation vector and $\mathbf{w}$ is a zero-mean white Gaussian vector with unknown variance. Because of the unknown parameters involved in the problem, no uniformly powerful test exists and the likelihood ratio test cannot be applied. A standard approach is then to resort to GLRT techniques that replace the unknown parameters with their maximum likelihood estimates. 
Let $\mathbf{H}_{i} \in \mathbb{R}^{N \times n_{i}}$ be a basis for the $n_{i}$-dimensional subspace $\mathcal{S}_{i}$ and let $\mathbf{P}_{\mathcal{S}_{i}}=\mathbf{H}_{i}\left(\mathbf{H}_{i}^{T} \mathbf{H}_{i}\right)^{-1} \mathbf{H}_{i}^{T}$ be its projection matrix. The GLRT for Problem (1) is a straightforward extension of the well-studied CFAR matched-subspace detector [1, Ch. 4] and can be written as [10]

$$
T_{\mathrm{GLRT}}(\mathbf{y})=\max _{1 \leq i \leq M} L_{i}(\mathbf{y}) \underset{\mathcal{H}_{0}}{\stackrel{\mathcal{H}_{1}}{\gtrless}} \eta_{\mathrm{GLRT}},
$$

where $\eta_{\mathrm{GLRT}}$ is the threshold chosen so as to satisfy some fixed test size. $L_{i}(\mathbf{y})$ is defined as twice the generalized loglikelihood ratio of $\mathbf{y}$ when $\mathbf{x}$ is assumed to belong to subspace $\mathcal{S}_{i}$. This ratio can be expressed as [16, pp. 372]

$$
L_{i}(\mathbf{y})=N \log \left(\frac{\mathbf{y}^{T} \mathbf{y}}{\mathbf{y}^{T}\left(\mathbf{I}_{N}-\mathbf{P}_{\mathcal{S}_{i}}\right) \mathbf{y}}\right)
$$

where $\mathbf{I}_{N}$ is the $N \times N$ identity matrix. As expressed in (2), the GLRT will always select the subspace containing the greatest amount of signal energy. As long as the subspaces have similar dimensions and/or are pairwise disjoint, this may be fine but in any other cases this is problematic. The worst case happens when the subspaces are nested, i.e., when $\mathcal{S}_{i} \subset \mathcal{S}_{\ell}$, for $1 \leq i<\ell \leq M$. In this scenario, the GLRT will always implement the test statistic with the matrix $\mathbf{P}_{\mathcal{S}_{M}}$ that projects the data in the larger subspace. If the actual signal subspace is smaller than $\mathcal{S}_{M}$, the GLRT will include noise-only samples under either hypothesis, degrading the performance of the detector. For instance, this problem is illustrated in [11] for a signal of unknown samples and unknown length corrupted by an additive white Gaussian noise with known variance. For this signal model, but now with an unknown noise variance, the GLRT (2) becomes a CFAR energy detector. More precisely, each basis satisfies

$$
\mathbf{H}_{i}=\left(\begin{array}{c}
\mathbf{I}_{n_{i}} \\
\mathbf{O}_{\left(N-n_{i}\right) \times n_{i}}
\end{array}\right)
$$

where $\mathbf{O}_{n \times m}$ is the $n \times m$ null matrix and $n_{i}<n_{\ell}$ for $i<$ $\ell$. The test statistic in (2) is then a monotonically increasing function of the ratio $\sum_{k=0}^{N-1}|y(k)|^{2} / \sum_{k=n_{M}}^{N-1}|y(k)|^{2}$, where $y(k)$ is the $k$-th entry of $\mathbf{y}$. If the actual signal length is smaller than $n_{M}$, the GLRT will not be effective since this ratio will be overestimated. More generally, this problem arises for any signal expressed as the sum of an unknown number of basis vectors.

Improvement of the GLRT can be achieved by using a model order selection criterion in the decision procedure. A well known criterion is the minimum description length (MDL) criterion [17], which leads to the following test ${ }^{1}$

$$
T_{\mathrm{MDL}}(\mathbf{y})=\max _{1 \leq i \leq M}\left\{L_{i}(\mathbf{y})-n_{i} \log N\right\} \underset{\mathcal{H}_{0}}{\stackrel{\mathcal{H}_{1}}{\gtrless}} \eta_{\mathrm{MDL}} .
$$

In [11], a multifamily likelihood ratio test (MFLRT) has also been proposed to accommodate nested models. It implements

${ }^{1}$ Other criteria such as AIC or GIC could also be considered [18]. the following decision rule:

$$
\begin{aligned}
T_{\operatorname{MFLRT}}(\mathbf{y})= & \\
\max _{1 \leq i \leq M}\left\{\left[L_{i}(\mathbf{y})-n_{i}\right.\right. & \left.\left(\log \left(\frac{L_{i}(\mathbf{y})}{n_{i}}\right)+1\right)\right] \\
& \left.\times u\left(\frac{L_{i}(\mathbf{y})}{n_{i}}-1\right)\right\} \underset{\mathcal{H}_{0}}{\stackrel{\mathcal{H}_{1}}{\gtrless}} \eta_{\text {MFLRT }},
\end{aligned}
$$

where $u(\cdot)$ is the unit step function. As illustrated in Sec. IV, by adding a penalty term, both tests (5) and (6) can provide a better performance than the GLRT. However, by expanding the scope of a new normalizing transformation introduced in [12], we will show that the performance can be further improved.

\section{MULTIFAMILY CFAR GLRT}

\section{A. Preliminary results}

To begin, we give some preliminary definitions and recall the main result of [12]. Let $K_{X}(t)$ be the cumulant generating function (CGF) of some random variable $X$, that is,

$$
K_{X}(t)=\log \mathbb{E}\left(e^{t X}\right)
$$

where $\mathbb{E}$ denotes the mathematical expectation. The Legendre transform (LT) of $K(\cdot)$ is then defined as

$$
K_{X}^{*}(x)=\sup _{t \in \mathcal{T}}\left(t x-K_{X}(t)\right),
$$

where $\mathcal{T}=\left\{t:\left|K_{X}(t)\right|<+\infty\right\}$. Next, define

$$
g_{X}(x)=2 K_{X}^{*}(x) u(X-\mathbb{E}(X)) .
$$

Proposition 1. [12] Let $X_{n}=\sum_{k=1}^{n} U_{k}$ where the $U_{k}$ 's are IID random variables with finite mean and variance. Also, assume that $\mathbb{E}\left(\left|U_{k}-\mathbb{E}\left(U_{k}\right)\right|^{3}\right)<\infty$. Define the random variable $Y_{n}$ as $Y_{n}=g_{X_{n}}\left(X_{n}\right)$. Then, $Y_{n} \stackrel{d}{\rightarrow} Y$, where the (generalized) probability density function (PDF) of $Y$ satisfies

$$
f_{Y}(y)=\left\{\begin{array}{ccrl}
\frac{1}{2 \sqrt{2 \pi y}} e^{-y / 2}, & y>0 \\
\frac{1}{2} \delta(y), & y=0 \\
0, & & \text { otherwise. }
\end{array}\right.
$$

This proposition states that, as $n$ tends to $+\infty, Y_{n}$ equals zero with probability $1 / 2$ and has a $\chi_{1}^{2}$ PDF with probability $1 / 2$. By applying a quadratic transformation to the standardized random variable $X_{n}$, a similar result could have been obtained by invoking the central limit-theorem (CLT). However, as illustrated in [12], the asymptotic regime can be reached faster than the CLT in some cases. Prop. 1 is relevant for nested detection problems as it can be used to equalize the modelorder dependent statistics involved in a GLRT solution. The function $g$, defined in (9), actually transforms the (asymptotic) GLR statistic to a $\chi_{1}^{2}$ random variable independent of the model order $n$. As shown in [12], if $L_{i}(\mathbf{y})$ can be expressed as the sum of $n_{i} \chi_{1}^{2}$ random variables under $\mathcal{H}_{0}$, then $g$ takes the form of the expression enclosed in braces in (6). The MLFRT (6) is thus a specific case of a more general theory resulting from the application of Prop. 1. Unfortunately, this proposition is not directly applicable to Problem (1) because the GLR statistic (3) is not expressed as a sum of independent random variables. However, by transforming (3), we will show that a similar result to that of Prop. 1 can be obtained for our specific problem. 


\section{B. Proposed detector}

Let $\tilde{L}_{i}(\mathbf{y})$ be the monotonically increasing function of $L_{i}(\mathbf{y})$ defined as

$$
\tilde{L}_{i}(\mathbf{y})=\frac{1}{2} \log \left(\frac{\mathbf{y}^{T} \mathbf{P}_{\mathcal{S}_{i}} \mathbf{y}}{\mathbf{y}^{T}\left(\mathbf{I}_{N}-\mathbf{P}_{\mathcal{S}_{i}}\right) \mathbf{y}} \times \frac{N-n_{i}}{n_{i}}\right) .
$$

The proposed detector is based on the derivation of a modelorder dependent penalty factor that results from the analysis of the LT-CGF of $\tilde{L}_{i}(\mathbf{y})$ under $\mathcal{H}_{0}$.

Under $\mathcal{H}_{0}, \tilde{L}_{i}(\mathbf{y})$ is half the logarithm of an F-distribution variate and therefore follows Fisher's z-distribution with $n_{i}$ and $N-n_{i}$ degrees of freedom. Let $\rho_{i}=N / n_{i}>1$, for $t<n_{i}\left(\rho_{i}-1\right)$, the CGF of $\tilde{L}_{i}(\mathbf{y})$ under $\mathcal{H}_{0}$ satisfies [19]

$$
\begin{array}{r}
K_{\tilde{L}_{i}}(t)=\frac{t}{2} \log \left(\rho_{i}-1\right)-\log \left(\Gamma\left(\frac{n_{i}\left(\rho_{i}-1\right)}{2}\right) \Gamma\left(\frac{n_{i}}{2}\right)\right) \\
+\log \left(\Gamma\left(\frac{n_{i}+t}{2}\right) \Gamma\left(\frac{n_{i}\left(\rho_{i}-1\right)-t}{2}\right)\right),
\end{array}
$$

where $\Gamma(z)=\int_{0}^{+\infty} t^{z-1} e^{-t} \mathrm{~d} t$. The LT of $K_{\tilde{L}_{i}}$ is then defined as $K_{\tilde{L}_{i}}^{*}(x)=\sup _{t<n_{i}\left(\rho_{i}-1\right)}\left(t x-K_{\tilde{L}_{i}}(t)\right)$ and

$$
g_{\tilde{L}_{i}}(x)=2 K_{\tilde{L}_{i}}^{*}(x) u\left(\tilde{L}_{i}(\mathbf{y})-\mu_{i}\right),
$$

where $\mu_{i}=\mathbb{E}\left(\tilde{L}_{i}(\mathbf{y})\right)=\frac{1}{2 n_{i}}\left(\frac{1}{\rho_{i}-1}-1\right)$.

Proposition 2. Let $Y_{n_{i}}$ be the random variable defined as $Y_{n_{i}}=g_{\tilde{L}_{i}}\left(\tilde{L}_{i}(\mathbf{y})\right)$ and assume that the ratio $\rho_{i}=N / n_{i}>1$ is kept constant as $n_{i} \rightarrow+\infty$. Under $\mathcal{H}_{0}, Y_{n_{i}} \stackrel{d}{\rightarrow} Y$, where the PDF of $Y$ is defined in Eq. (10).

Proof: See Appendix A.

Applying the transformation $g_{\tilde{L}_{i}}$ to the statistic $\tilde{L}_{i}(\mathbf{y})$ is therefore a way to equalize the model-order dependent PDFs involved in our problem. Unfortunately, $g_{\tilde{L}_{i}}\left(\tilde{L}_{i}(\mathbf{y})\right)$ has no closed-form expression. However, based on the asymptotic analysis provided in App. B, we are in position to formulate an explicit approximation of $g_{\tilde{L}_{i}}\left(\tilde{L}_{i}(\mathbf{y})\right)$, that is

$$
\begin{aligned}
g_{\tilde{L}_{i}}\left(\tilde{L}_{i}(\mathbf{y})\right) \approx 2\left[\tilde{L}_{i}(\mathbf{y}) h_{i}\left(\tilde{L}_{i}(\mathbf{y})\right)\right. & \left.-K_{\tilde{L}_{i}}\left(h_{i}\left(\tilde{L}_{i}(\mathbf{y})\right)\right)\right] \\
& \times u\left(\tilde{L}_{i}(\mathbf{y})-\mu_{i}\right),
\end{aligned}
$$

where

$$
h_{i}(x)=n_{i}\left(\frac{\rho_{i}}{\left(\rho_{i}-1\right) e^{-2 x}+1}-1\right) .
$$

Finally, to solve Problem (1), we propose the following test, referred to as the MFCLRT,

$$
T_{\text {MFCLRT }}(\mathbf{y})=\max _{1 \leq i \leq M}\left\{g_{\tilde{L}_{i}}\left(\tilde{L}_{i}(\mathbf{y})\right)\right\} \underset{\mathcal{H}_{0}}{\stackrel{\mathcal{H}_{1}}{\gtrless}} \eta_{\text {MFCLRT }},
$$

where the approximation (14) is used for $g_{\tilde{L}_{i}}$. This test does not depend on unknown parameters and therefore ensures the CFAR property. Moreover, it can be shown that $g_{\tilde{L}_{i}}\left(\tilde{L}_{i}(\mathbf{y})\right)$ is a decreasing function of $n_{i}$ so that it counteracts the tendency of the GLR statistic to increase with $n_{i}$. Also, note that if the subspace dimensions are all identical $\left(n_{i}=n, \forall i\right)$, then the tests (2), (5), (6) and (16) are all equivalent since their statistics are all monotonically increasing with $L_{i}(\mathbf{y})$.

\section{NumericAl Results}

To illustrate the performance of the MFCLRT (16), we consider the detection of a known signal $\mathbf{s}$ in an unknown deterministic multipath channel $\mathbf{h}$. More precisely, $x(k)=$ $\sum_{l=0}^{n_{M}-1} h(l) s(k-l)$, where $n_{M}$ denotes the number of channel taps. In the simulation, the channel has an exponential decay power-delay profile, i.e., $h(k)=e^{-k \frac{\log (10)}{2 k_{0}}}$, where $k_{0}>0$ denotes the $10 \mathrm{~dB}$-maximum delay spread. ${ }^{2}$ Without loss of generality, we assume that $s(k)=d(k)$, where $d(k)$ is the unit impulse. ${ }^{3}$ Since the channel is unknown, the detector is unaware of the number of significant echoes that must be taken into account in the test statistic. The set of all possible echoes therefore constitutes a union of nested subspaces, each of which corresponds to a set of delays. More precisely, since $s(k)=d(k)$, each subspace basis is defined as in Eq. (4) for any $1 \leq n_{i} \leq n_{M}$.

We investigate the performance of the proposed detector in terms of probability of detection $P_{d}$. The probability of false alarm $P_{f a}$ is set to $10^{-3}$. The detection thresholds and the probabilities of detection are obtained with $100 / P_{f a}$ and $10^{4}$ independent trials, respectively. The signal length $N$ is set to 100 and $n_{M}=50$. The energy-to-noise ratio (ENR) is defined as $\mathrm{ENR}=10 \log _{10}\left(\mathbf{x}^{T} \mathbf{x} / \sigma^{2}\right)$, where $\sigma^{2}$ is the noise variance.

Fig. 1 shows the probability of detection as function of the ENR for the four tests (2), (5), (6) and (16). $k_{0}$ is set to 15. The MFCLRT clearly outperforms the other tests as it better estimates the order of the channel. The GLRT includes all the echoes in the statistic, even those with insignificant power, and therefore performs the worst. A detailed analysis (not shown here) reveals that the MDL test tends to only consider the first few powerful echoes, whereas the MFLRT often underpenalizes insignificant echoes.

The effect of the model order is analyzed in Fig. 2 by varying the value of the maximum delay spread $k_{0}$. Once again, the MFCLRT outperforms the other tests for any value $k_{0}$. Both the MFCLRT and the MFLRT converge to the performance of the GLRT as $k_{0}$ increases. This results from the increase in the number of significant echoes as $k_{0}$ increases, leading to a model order close to the maximum $n_{M}$. Surprisingly, a collapse of performance rapidly occurs for the MDL test. This can be explained by the penalty term in Eq. (5) that linearly increases with $n_{i}$ so that the channel tail is too often ignored.

\section{CONCLUSiON}

We have shown that existing methods, such as the GLRT, are not adapted to the detection of signals in a union of possibly nested subspaces. By adapting to our context the CGF-based transformation presented in [12], we have derived a new detector capable of equalizing the model-order dependent statistics. Although the equalization is of an asymptotic nature (as the subspace dimension becomes large), the resulting test turns out to be very efficient, even for low-dimensional subspaces.

\footnotetext{
${ }^{2}$ We recall that this is the time delay (in number of samples) after which the multipath power falls $10 \mathrm{~dB}$ below the maximum.

${ }^{3} s(k)$ can be considered as the output of a correlation-based channel sounder for instance.
} 


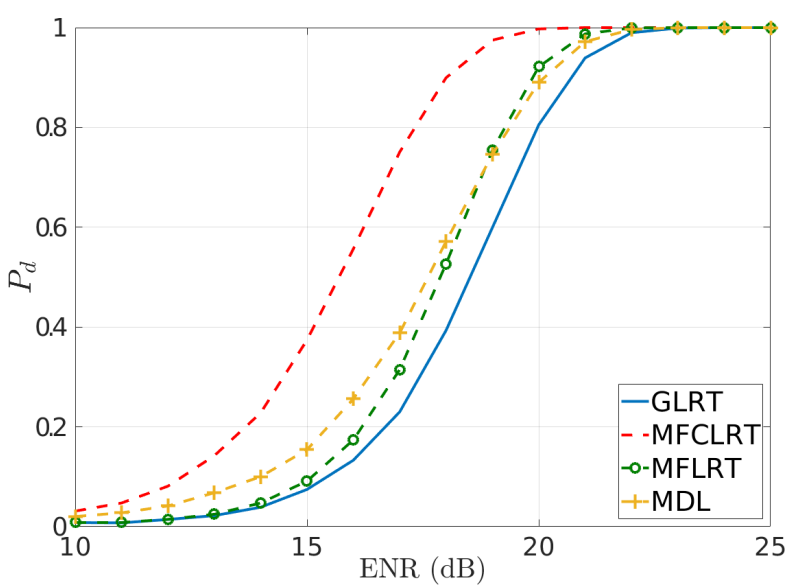

Fig. 1. $P_{d}$ as a function of ENR for the GLRT, the MDL test, the MFLRT and the MFCLRT. $P_{f a}=10^{-3}, N=100, n_{M}=50, k_{0}=15$.

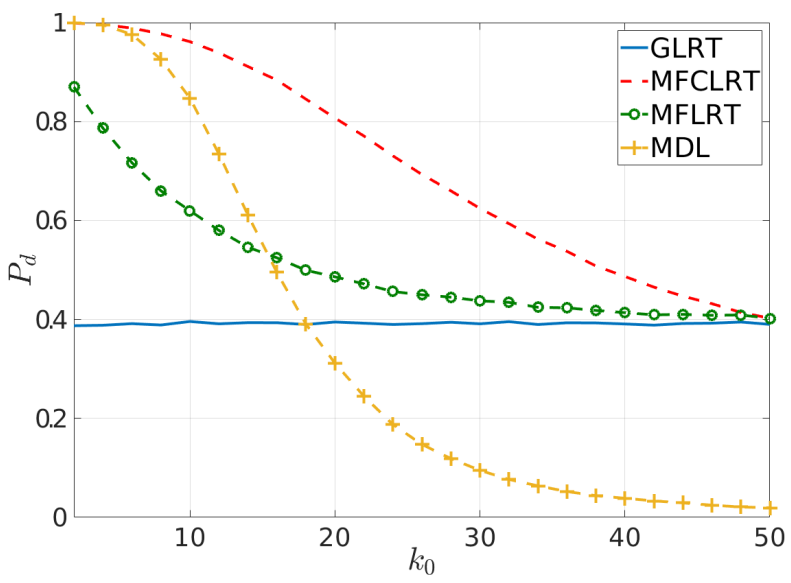

Fig. 2. $P_{d}$ as a function of $k_{0}$ for the GLRT, the MDL test, the MFLRT and the MFCLRT. $P_{f a}=10^{-3}, N=100, n_{M}=50$, ENR $=18 \mathrm{~dB}$.

\section{APPENDiX A}

\section{PROOF OF PROP. 2}

Let $\mu_{i}$ and $\sigma_{i}^{2}$ denote the expectation and the variance of the random variable $\tilde{L}_{i}(\mathbf{y})$ under $\mathcal{H}_{0}$. Since $\tilde{L}_{i}(\mathbf{y})$ follows Fisher's z-distribution, we have $\mu_{i}=\frac{1}{2 n_{i}}\left(\frac{1}{\rho_{i}-1}-1\right)$ and $\sigma_{i}^{2}=\frac{1}{2 n_{i}}\left(\frac{1}{\rho_{i}-1}+1\right)$. Using a Taylor expansion for the LTCGF about the expectation $\mu_{i}$, we have [12, App. B]

$$
\begin{aligned}
& g_{\tilde{L}_{i}}\left(\tilde{L}_{i}(\mathbf{y})\right)=u\left(\tilde{L}_{i}(\mathbf{y})-\mu_{i}\right) \times \\
& {\left[\frac{\left(\tilde{L}_{i}(\mathbf{y})-\mu_{i}\right)^{2}}{\sigma_{i}^{2}}+\frac{1}{3} K_{\tilde{L}_{i}}^{*^{(3)}}\left(\xi_{i}\right) \sigma_{i}^{3} \frac{\left(\tilde{L}_{i}(\mathbf{y})-\mu_{i}\right)^{3}}{\sigma_{i}^{3}}\right],}
\end{aligned}
$$

where $\xi_{i}=\mu_{i}+\theta\left(\tilde{L}_{i}(\mathbf{y})-\mu_{i}\right)$ and $0<\theta<1$. It is known that the distribution of a Fisher's $\mathrm{z}$ variable approaches normality when its degrees of freedom tends to infinity [19, Sec. 15]. More precisely, let $V_{n_{i}}=\frac{\tilde{L}_{i}(\mathbf{y})-\mu_{i}}{\sigma_{i}}$. For any $\rho_{i}=N / n_{i}>1$, $V_{n_{i}} \stackrel{d}{\rightarrow} \mathcal{N}(0,1)$. Therefore, by the continuous mapping theorem, $V_{n_{i}}^{2} \stackrel{d}{\rightarrow} \chi_{1}^{2}$. The main step of the proof is then to show that the second term on the r.h.s of (17) is bounded in probability by a decreasing function of $n_{i}$.
First, since $\sigma_{i}^{2}$ is finite, then [15, Th. 14.4-1] $\left(\tilde{L}_{i}(\mathbf{y})-\mu_{i}\right)^{3} / \sigma_{i}^{3}=O_{p}(1)$. In addition, using [12, App. A], we note that the third derivative of the LT-CGF satisfies

$$
K_{\tilde{L}_{i}}^{*^{(3)}}\left(\xi_{i}\right)=-\frac{K_{\tilde{L}_{i}}^{(3)}\left(t_{i}^{*}\left(\xi_{i}\right)\right)}{\left(K_{\tilde{L}_{i}}^{(2)}\left(t_{i}^{*}\left(\xi_{i}\right)\right)\right)^{3}},
$$

where $t_{i}^{*}(x)=\operatorname{argsup}_{t<n_{i}\left(\rho_{i}-1\right)}\left(t x-K_{\tilde{L}_{i}}(t)\right)$. Based on the expression of the CGF defined in (12), we observe that the ratio (18) will depend on the $3^{\text {rd }}$ and $2^{\text {nd }}$-order derivative of the logarithm of the gamma function. These derivatives are known as the polygamma functions of order 2 and 1 [20, Ch. 6.4], respectively. Based on the asymptotic expansion given [20, Eq. (6.4.11)], we deduce that the $m$-th order polygamma function $\Psi^{(m)}(z)$ satisfies $\Psi^{(m)}(z)=O\left(z^{-m}\right)(z \rightarrow \infty)$. Moreover, as shown in App. B, $\forall x>0, t_{i}^{*}(x)$ is strictly increasing in $n_{i}$. Consequently, $\forall x>0$,

$$
\frac{K_{\tilde{L}_{i}}^{(3)}\left(t_{i}^{*}(x)\right)}{\left(K_{\tilde{L}_{i}}^{(2)}\left(t_{i}^{*}(x)\right)\right)^{3}}=O\left(\frac{\Psi^{(2)}\left(n_{i}\right)}{\left(\Psi^{(1)}\left(n_{i}\right)\right)^{3}}\right)=O\left(n_{i}\right) .
$$

In addition, provided that $\tilde{L}_{i}(\mathbf{y})>\mu_{i}, \xi_{i}$ is strictly positive. Therefore, based on Eqs (18) and (19), we get $K_{\tilde{L}_{i}}^{*(3)}\left(\xi_{i}\right)=$ $O_{p}\left(n_{i}\right)$, so that

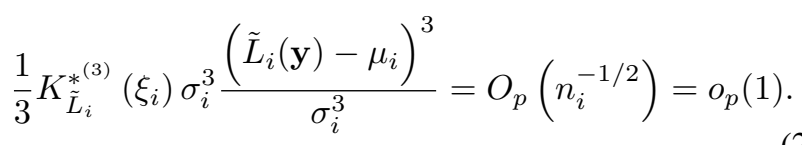

In summary, $g_{\tilde{L}_{i}}\left(\tilde{L}_{i}(\mathbf{y})\right)=u\left(V_{n_{i}}\right) V_{n_{i}}^{2}+o_{p}(1)$ with $V_{n_{i}} \stackrel{d}{\rightarrow}$ $\mathcal{N}(0,1)$. Therefore, according to the continuous mapping theorem and to [15, Th. 4.4-3], $g_{\tilde{L}_{i}}\left(\tilde{L}_{i}(\mathbf{y})\right)$ converges in distribution to the random variable $Y$ whose PDF is expressed in Eq. (10).

\section{APPENDIX B ASYMPTOTICS OF $t_{i}^{*}(x)$}

Let $t_{i}^{*}(x)$ be defined as $t_{i}^{*}(x)=\operatorname{argsup}_{t<n_{i}\left(\rho_{i}-1\right)} G_{i}(t, x)$ where $G_{i}(t, x)=\left(t x-K_{\tilde{L}_{i}}(t)\right)$. From (12), we deduce that

$$
\begin{aligned}
\frac{\partial G_{i}(t, x)}{\partial t}=\frac{1}{2}\left[2 x-\log \left(\rho_{i}-1\right)-\Psi^{(0)}\left(\frac{1}{2}\left(n_{i}+t\right)\right)\right. \\
\left.+\Psi^{(0)}\left(\frac{1}{2}\left(n_{i}\left(\rho_{i}-1\right)-t\right)\right)\right],
\end{aligned}
$$

where $\Psi^{(0)}(\cdot)$ is the digamma function. For any real variable $z$, this function satisfies [20, Eq. (6.3.18)]: $\Psi^{(0)}(z)=\log (z)+$ $o\left(z^{-1}\right)$. Therefore, as $n_{i} \rightarrow \infty$, we have

$$
\frac{\partial G_{i}(t, x)}{\partial t} \underset{n_{i} \rightarrow \infty}{\sim} \frac{1}{2}\left[2 x+\log \left(\frac{\left.n_{i}-t /\left(\rho_{i}-1\right)\right)}{n_{i}+t}\right)\right] .
$$

By setting the derivative to zero, we can conclude that

$$
t_{i}^{*}(x) \underset{n_{i} \rightarrow \infty}{\sim} n_{i}\left(\frac{\rho_{i}}{\left(\rho_{i}-1\right) e^{-2 x}+1}-1\right) .
$$

Note that $\left.\frac{\partial^{2} G_{i}(t, x)}{\partial^{2} t}\right|_{t=t_{i}^{*}(x)}<0, \forall x$, so that $t_{i}^{*}(x)$ is an (asymptotic) supremum. Also, observe that $\forall x>0, t_{i}^{*}(x)$ is strictly increasing in $n_{i}$. 


\section{REFERENCES}

[1] L. L. Scharf, Statistical Signal Processing: Detection, Estimation, and Time Series Analysis, Addison-Wesley, 1991.

[2] S. Kraut and L. L. Scharf, "The CFAR adaptive subspace detector is a scale-invariant GLRT," IEEE Transactions on Signal Processing, vol. 47, no. 9, pp. 2538 - 2541, September 1999.

[3] S. Kraut, L. L. Scharf, and L. T. McWhorter, "Adaptive Subspace Detectors," IEEE Transactions on Signal Processing, vol. 49, no. 1, pp. 1 - 16, Jan. 2001.

[4] Y. Jin and B. Friedlander, "A CFAR Adaptive Subspace Detector for Second-Order Gaussian Signals," IEEE Transactions on Signal Processing, vol. 53, no. 3, pp. 871 - 884, Mar. 2005.

[5] F.-X. Socheleau and D. Pastor, "Testing the energy of random signals in a known subspace: An optimal invariant approach," IEEE Signal Processing Letters, vol. 21, no. 10, pp. 1182-1186, 2014.

[6] D. Pastor and F.-X. Socheleau, "Random distortion testing with linear measurements," Signal Processing, vol. 145, pp. 116-126, 2018.

[7] Y. M. Lu and M. N. Do, "A theory for sampling signals from a union of subspaces," IEEE Transactions on Signal Processing, vol. 56, no. 6, pp. 2334-2345, 2008.

[8] F. Gini, M. Greco, and F. Farina, "Radar detection and preclassification based on multiple hypothesis," IEEE transactions on aerospace and electronic systems, vol. 40, no. 3, pp. 1046-1059, 2004.

[9] T. Wimalajeewa, Y. C. Eldar, and P. K. Varshney, "Subspace recovery from structured union of subspaces," IEEE Transactions on Information Theory, vol. 61, no. 4, pp. 2101-2114, 2015.

[10] M. A. Lodhi and W. U. Bajwa, "Detection theory for union of subspaces," IEEE Transactions on Signal Processing, vol. 66, no. 24 pp. 6347-6362, 2018.
[11] S. Kay, "The multifamily likelihood ratio test for multiple signal model detection," IEEE Signal Processing Letters, vol. 12, no. 5, pp. 369-371, 2005.

[12] S. Kay and Y. Rawashdeh, "A new random variable normalizing transformation with application to the GLRT," IEEE Signal Processing Letters, vol. 25, no. 2, pp. 189-193, 2018.

[13] A. De Maio, S. De Nicola, and A. Farina, "GLRT versus MFLRT for adaptive CFAR radar detection with conic uncertainty," IEEE Signal Processing Letters, vol. 16, no. 8, pp. 707 - 710, 2009.

[14] O. Harel and H. Messer, "Extension of the MFLRT to detect an unknown deterministic signal using multiple sensors, applied for precipitation detection," IEEE Signal Processing Letters, vol. 20, no. 10, pp. 945-948, 2013.

[15] Y. M. Bishop, S. E. Fienberg, and P. W. Holland, Discrete multivariate analysis: theory and practice, Springer Science \& Business Media, 2007.

[16] S. M. Kay, Fundamentals of Statistical Signal Processing, Volume II, Detection Theory, 14th printing, Prenctice Hall, 2009.

[17] J. Rissanen, "Modeling by shortest data description," Automatica, vol. 14, no. 5, pp. 465-471, 1978.

[18] P. Stoica and Y. Selen, "Model-order selection: a review of information criterion rules," IEEE Signal Processing Magazine, vol. 21, no. 4, pp. 36-47, 2004.

[19] L. A Aroian, "A study of RA Fisher's z distribution and the related F distribution," The Annals of Mathematical Statistics, vol. 12, no. 4, pp. 429-448, 1941.

[20] M. Abramowitz and I. Stegun, Handbook of Mathematical Functions. Ninth printing., Dover Publications Inc., New York, 1972. 